\title{
Makrofotografie mit der Stacking-Technik am Beispiel von Nadelgehölz-Zapfen
}

\author{
Peter König \& Michael Luhn
}

\section{Abstract}

Conventional macro shots often lack depth of field. A novel digital process („stacking“) takes photographs in sections and joins the sharp portions of the images computationally, so that the subjects are reproduced sharply from front to back. The photographically achievable results of this technique are presented by using examples of conifer flower structures.

\section{Zusammenfassung}

Herkömmlichen Makroaufnahmen mangelt es häufig an Tiefenschärfe. Ein neuartiges digitales Verfahren („Stacking“) nimmt Objekte abschnittsweise auf und fügt die scharfen Anteile der Bilder rechentechnisch zusammen, so dass die Motive von vorn bis hinten scharf wiedergegeben werden. Die fotografisch erzielbaren Resultate dieser Technik werden am Beispiel von Nadelgehölzblüten vorgestellt.

\section{Problemstellung und Methodik}

Jeder, der sich schon mal in das Gebiet der Makrofotografie vorgewagt hat, ist meist von den Möglichkeiten begeistert, aber auch schnell wieder frustriert. Das liegt entweder an den oft unscharfen Bildern, die man verwackelt hat oder den Fokus nicht an die gewollte Stelle bekam. Dieses Problem lässt sich allerdings mit Stativ, Makroschlitten und Blitzlicht umgehen. Ein oft viel größeres Manko ist die mit steigendem Abbildungsmaßstab kleiner werdende Tiefenschärfe. Natürlich könnte man nun sagen: Okay, dann blende ich auf f/32 ab und gut ist. Bei den meisten Objektiven tritt aber schon beim Abblenden auf f/11 oder gar f/8 eine mehr

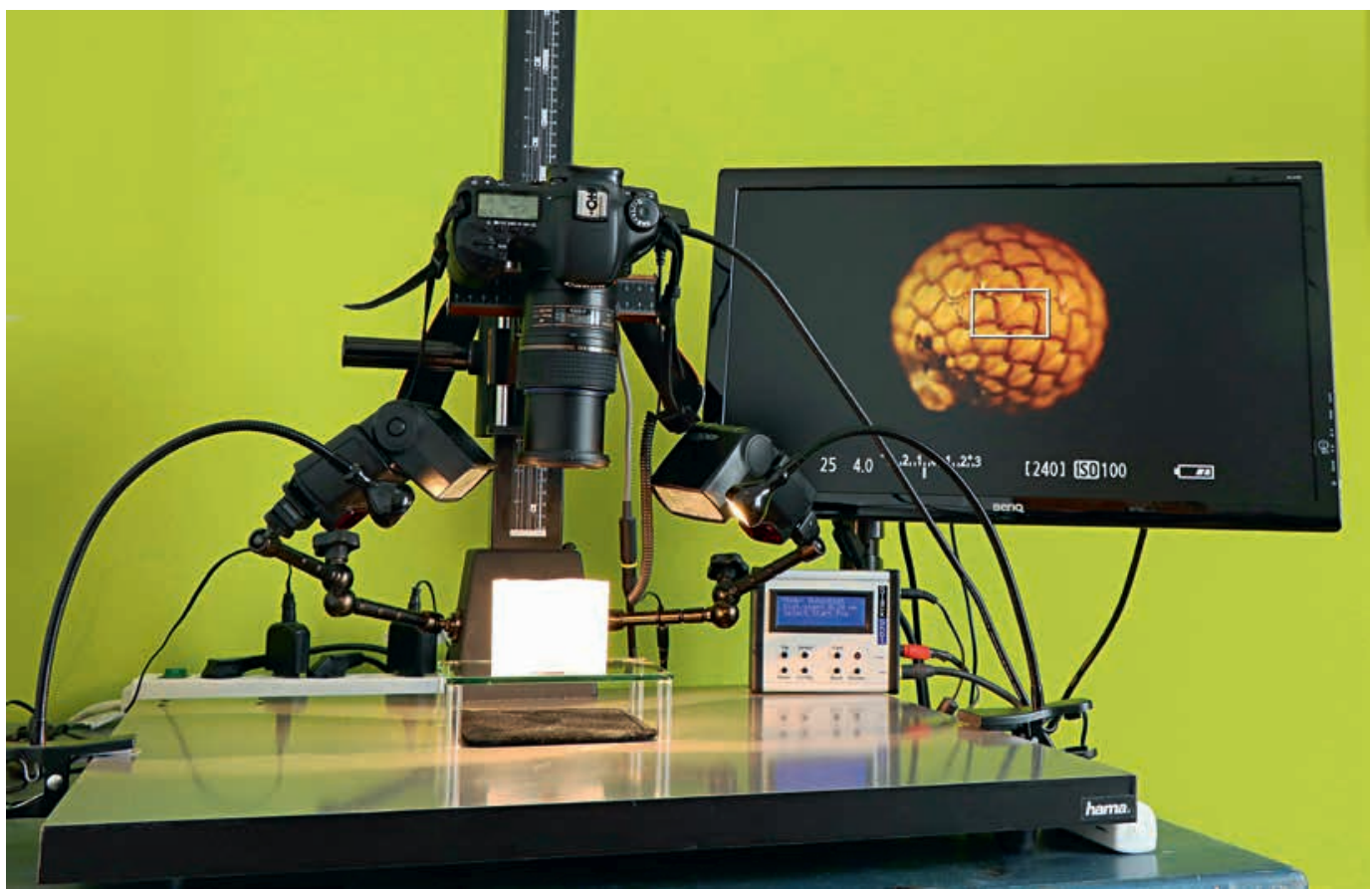

Abb. 1: Die Spiegelreflexkamera mit dem Makroobjektiv ist auf einem Linearvorschub der Fa. Cognysis montiert. Der Mikrocontroller „Stackshot“ steuert das Timing, den Vorschub und löst die Kamera aus. Die beiden LED-Lampen dienen als Einstelllicht und werden während der Aufnahme mit dem Blitzlicht ausgeschaltet. 
oder minder deutlich wahrnehmbare Beugungsunschärfe auf. Die Bilder werden einfach nicht mehr „knackig“.

Fotoobjektive haben ihr größtes Auflösungsvermögen, wenn man sie um ein bis zwei Blendenstufen abblendet. Dennoch beträgt die Tiefenschärfe dann bei einem $90 \mathrm{~mm}$ Makroobjektiv, einem Abbildungsmaßstab von 1:1 und einer Blende von $\mathrm{f} / 4$ gerade mal etwa $1 \mathrm{~mm}$. In manchen Fällen ist das ausreichend oder gar erwünscht, wenn bei einer Blüte nur die Staubblätter scharf abzubilden sind und der Rest der Blume sich weich und unscharf nach hinten fortsetzt. Aber für die detailgetreue Wiedergabe sollte möglichst Alles von vorn bis hinten scharf und mit hoher Detailaufösung dargestellt werden.

Hier setzt nun die Idee des Stapelns (engl. stacking) mehrerer Bilder an. Dazu braucht man im einfachsten Fall ein unbewegliches Motiv und einen Makroschlitten. Wenn eine Motivtiefe von $20 \mathrm{~mm}$ scharf abgebildet werden soll, benötigt man im oben genannten Beispiel etwa 27 Bilder (ein Drittel Überlappung sollte man in der Tiefe schon haben). Aus jedem Bild dieses Stapels berechnet nun eine spezielle Software die scharfen Bereiche in diesem Bild und speichert sie ab. Zum Schluss werden alle diese Anteile miteinander verrechnet (übereinandergelegt) und das Ergebnis ist ein von vorn bis hinten scharfes Bild.

\section{Vorgehensweise}

Einem guten Bild geht eine aufwändige Reinigung des Objektes unter dem Stereomikroskop voraus. Denn Staubkrümel und Fussel lenken besonders gut vom eigentlichen Motiv ab. Als Lichtquelle sind mindestens zwei Blitzgeräte mit Diffusoren zu empfehlen, da die normalen weißen LEDs nur einen recht mäßigen Farbwiedergabeindex haben und sich die Belichtungszeit im Vergleich zum Blitzen stark erhöht. Die im Folgenden abgebildeten Koniferen wurden mit einer Canon EOS 7D und einem Makroobjektiv $90 \mathrm{~mm} / 2,8$ von Tamron aufgenommen. Zusätzlich wurde ein $\mathrm{Li}$ nearvorschub mit Controller von der Firma Cognysis (USA) benutzt, der das Arbeiten doch sehr vereinfacht: man stellt Anfangs- und Endpunkt ein, gibt die Schrittweite vor und los geht's. Bei den kleinen Details reichten durchaus 30 Bilder, aber für die tiefen Übersichtsaufnahmen waren es auch schon mal über 100 Bilder. Schon für ein kleines Ausstellungsprojekt wird über $1000 \mathrm{Mal}$ der Auslöser betätigt.

Hinzu kommt, dass sich frisch gepflückte Pflanzen während dieser Prozedur doch bewegen können und den Bilderstapel dadurch verderben. Bei einigen Aufnahmen „rannte“ auch schon mal die eine oder andere Milbe durch das Bild. Insofern gilt die Empfehlung, erst einmal mit einem nicht glänzenden Steinchen als Motiv anzufangen. Als Stapel-Software kommen verschiedene Programme in Frage: „Zerene Stacker“, „Helicon Focus“, „CombineZM“ oder „Picolay“ (das erste wurde hier favorisiert, die beiden letzten sind Freeware). Die nachfolgende Bildoptimierung kann dann mit „Adobe Photoshop“ oder „GIMP“ vorgenommen werden.

\section{Die Zapfen der Nadelgehölze}

Die Zapfen der Nadelgehölze sind zumeist klein oder unscheinbar. Da die Bestäubung durch den Wind erfolgt, fehlen in den meisten Fällen eine auffällige Gestalt und Färbung. Über den Aufbau und ihre Bestäubungsbiologie berichteten STÜTZEL \& Röweкамp (1997) und Jagel (2011) in dieser Zeitschrift. Dörken \& Nimsch (2019) geben eine Koniferen-Zusammenstellung der Erde. Die Makrofotografie in Stacking-Technik bietet sich an, die Objekte für den Betrachter heranzuzoomen und quasi dreidimensional darzustellen.

Die Zapfen der Koniferen sind ferner eingeschlechtig ausgebildet; damit wird die Gefahr einer Selbstbefruchtung reduziert. Die Geschlechtsverteilung ist zumeist einhäusig, also männliche ( $\left.\sigma^{\top}\right)$ und weibliche Zapfen (ㅇ) auf einer Pflanze, seltener zweihäusig (etwa bei den Eiben) ausgebildet.

Die männlichen Zapfen bestehen aus den Staubblättern, die Pollen (Blütenstaub) in enormen Menge produzieren, damit wenigstens einige Körner die zugehörigen weiblichen Zapfen und die darin ausgebildeten Samenanlagenerreichen. 
Die weiblichen Zapfen bestehen aus einer zentralen Spindel, an der in den Achseln von sterilen Deckschuppen (= Tragblätter) jeweils die Samenschuppen angeordnet sind. Auf diesen werden die Samen, die zur Reife ein, zwei oder mehr Jahre benötigen, gebildet. Bei zahlreichen Arten dient ein Bestäubungstropfen dem Pollenfang. Da die Samen nicht wie bei den Bedecktsamern von einem Fruchtblatt umhüllt sind, sondern frei dem Luftraum ausgesetzt sind, spricht man von Nacktsamern.

Die Samen sind häufig geflügelt, die Verbreitung übernimmt entsprechend der Wind. Bei der Eibe und ihren Verwandten stehen die äußer- lich kaum sichtbar verholzten weiblichen Zapfen meist einzeln, ihre Samen sind von einem auffällig gefärbten Samenmantel umgeben. Hier sorgen Vögel für die Ausbreitung des Nachwuchses.

Für die nachstehend wiedergegebenen Motive wurde eine samtschwarze Rückseite gewählt, um den Farbkontrast hervorzuheben und keinen störenden Hintergrund zu haben. Die Bilder stellen einen Auszug einer Fotoausstellung dar, die 2018 im Botanischen Garten Greifswald gezeigt wurde. Viel Spaß beim Betrachten!

\subsection{Pinaceae - Kieferngewächse}
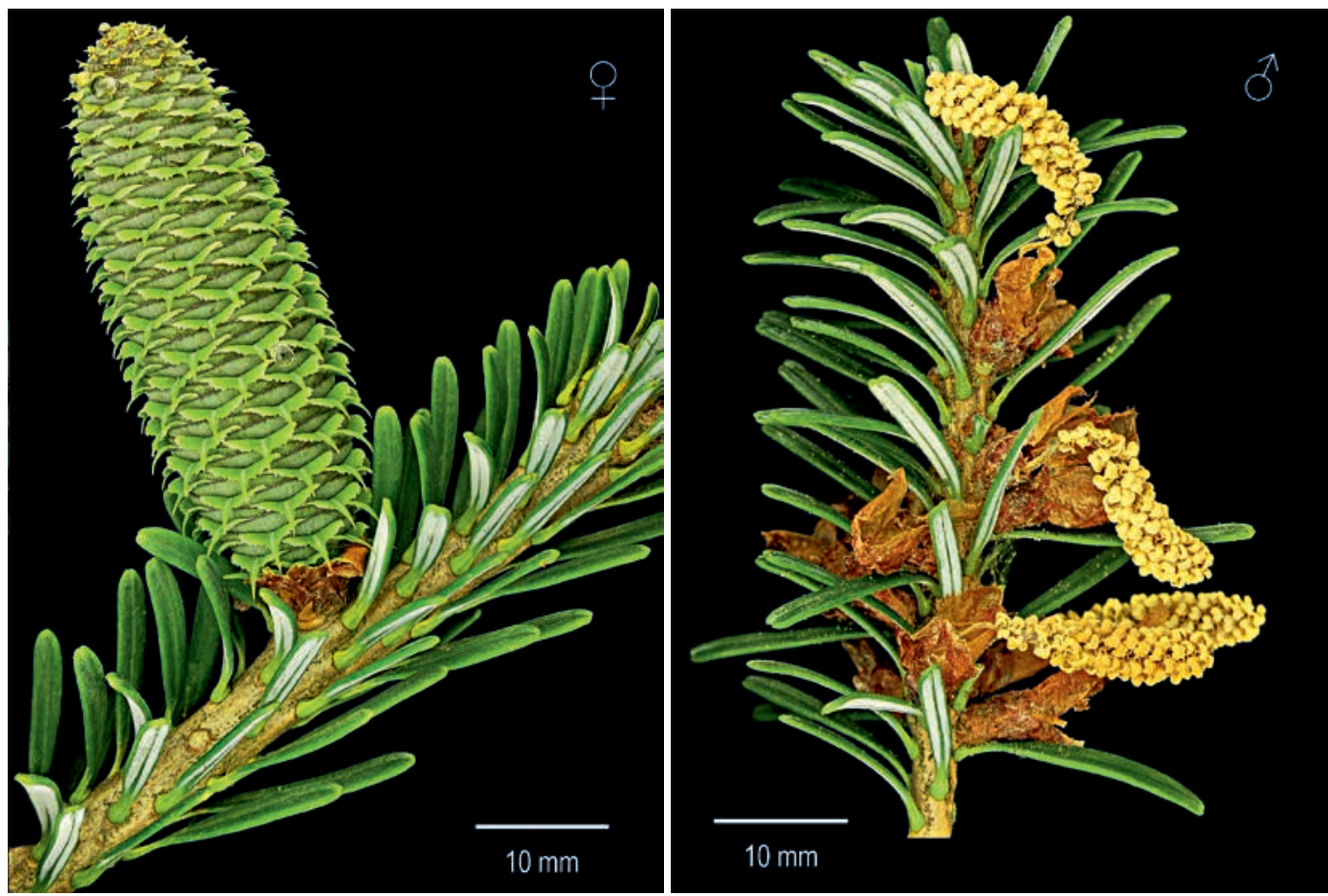

Abb. 2: Bei der Koreanischen Tanne (Abies koreana) kontrastieren die breiten, silberweißen Spaltöffnungsstreifen mit dem Nadelgrün und den gelblichen männlichen Zapfen mit den verbliebenen braunen Knospenschuppen. Die weiblichen Zapfen bleiben bis zur Reife mehr oder weniger aufrecht stehen; die Deckschuppen ragen etwas heraus, ihre Spitzen sind nach unten umgeschlagen. 


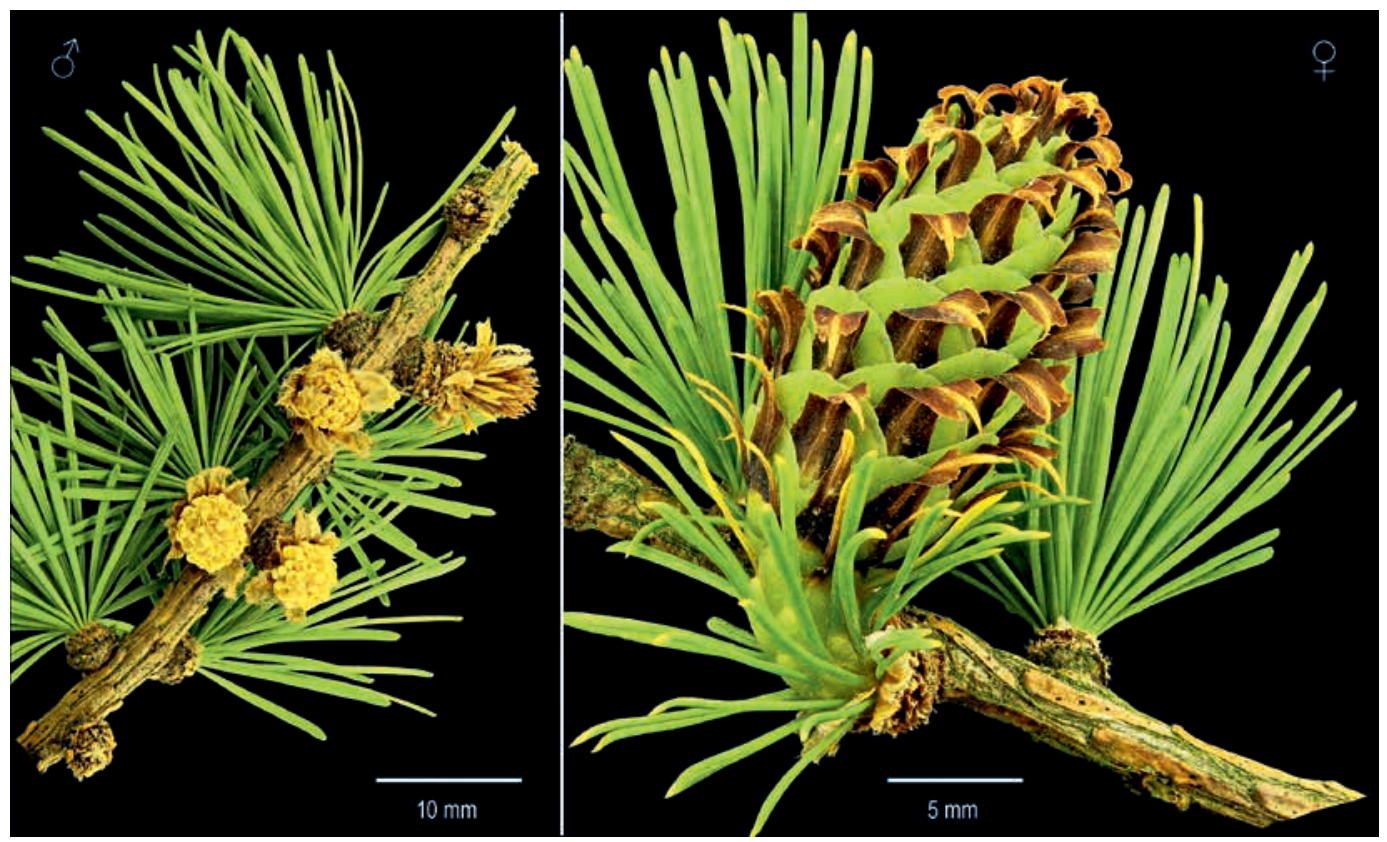

Abb. 3: Prinz-Rupprecht-Lärche (Larix gmelinii var. principis-rupprechtii). Im heranwachsenden weiblichen Zapfen heben sich die beige-braunen Deck- und die grünlichen Samenschuppen deutlich ab.

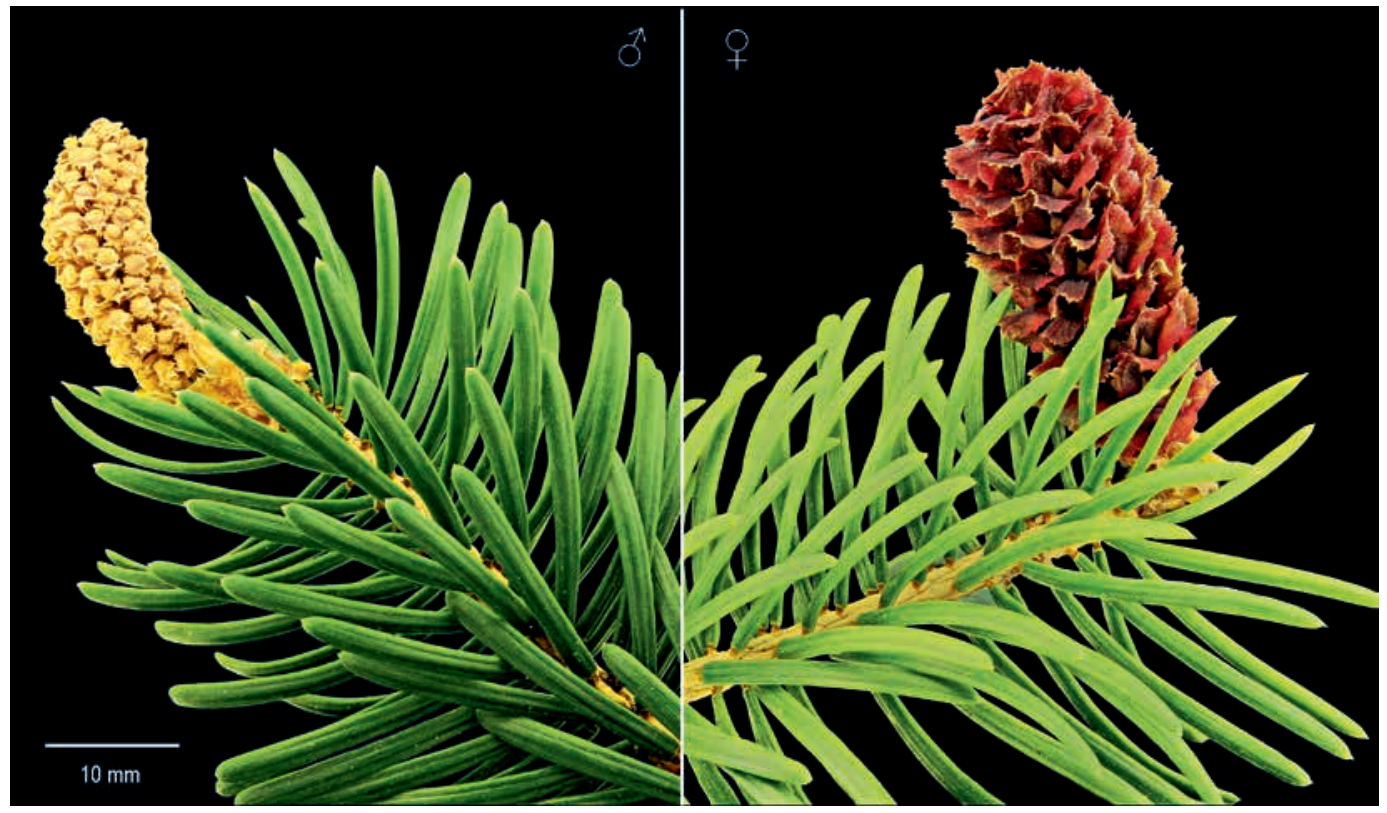

Abb. 4: Yedo-Fichte (Picea jezoensis). Auffällig sind die rötlich-braun heranwachsenden weiblichen Zapfen, die ein auffälliges Farbspiel mit dem Grün der Nadeln zeigen. 


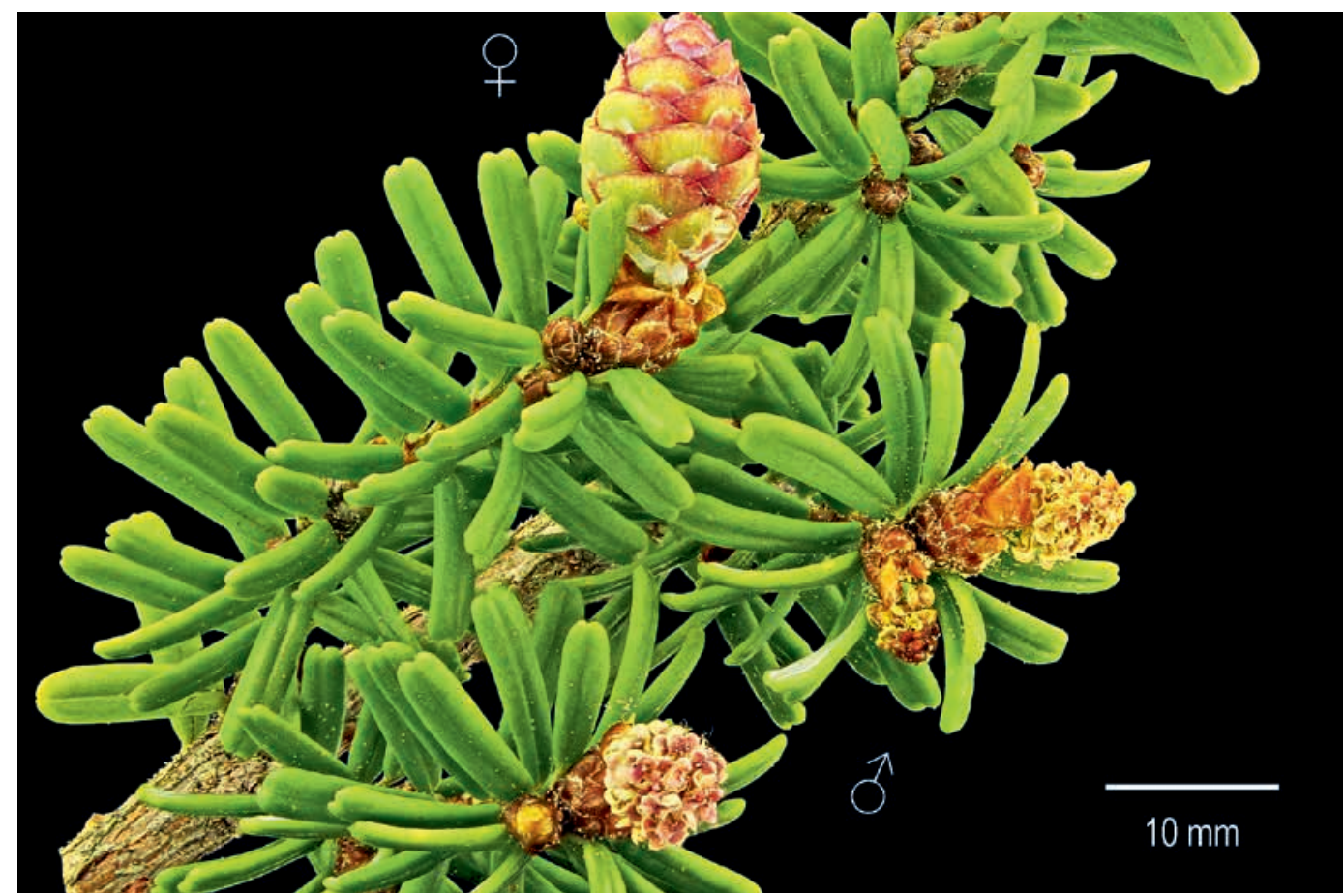

Abb. 5: Nordjapanische Hemlocktanne (Tsuga diversifolia). Die weiblichen Zapfen erreichen ausgewachsen eine Länge von 25 mm.

\subsection{Cupressaceae - Zypressengewächse}

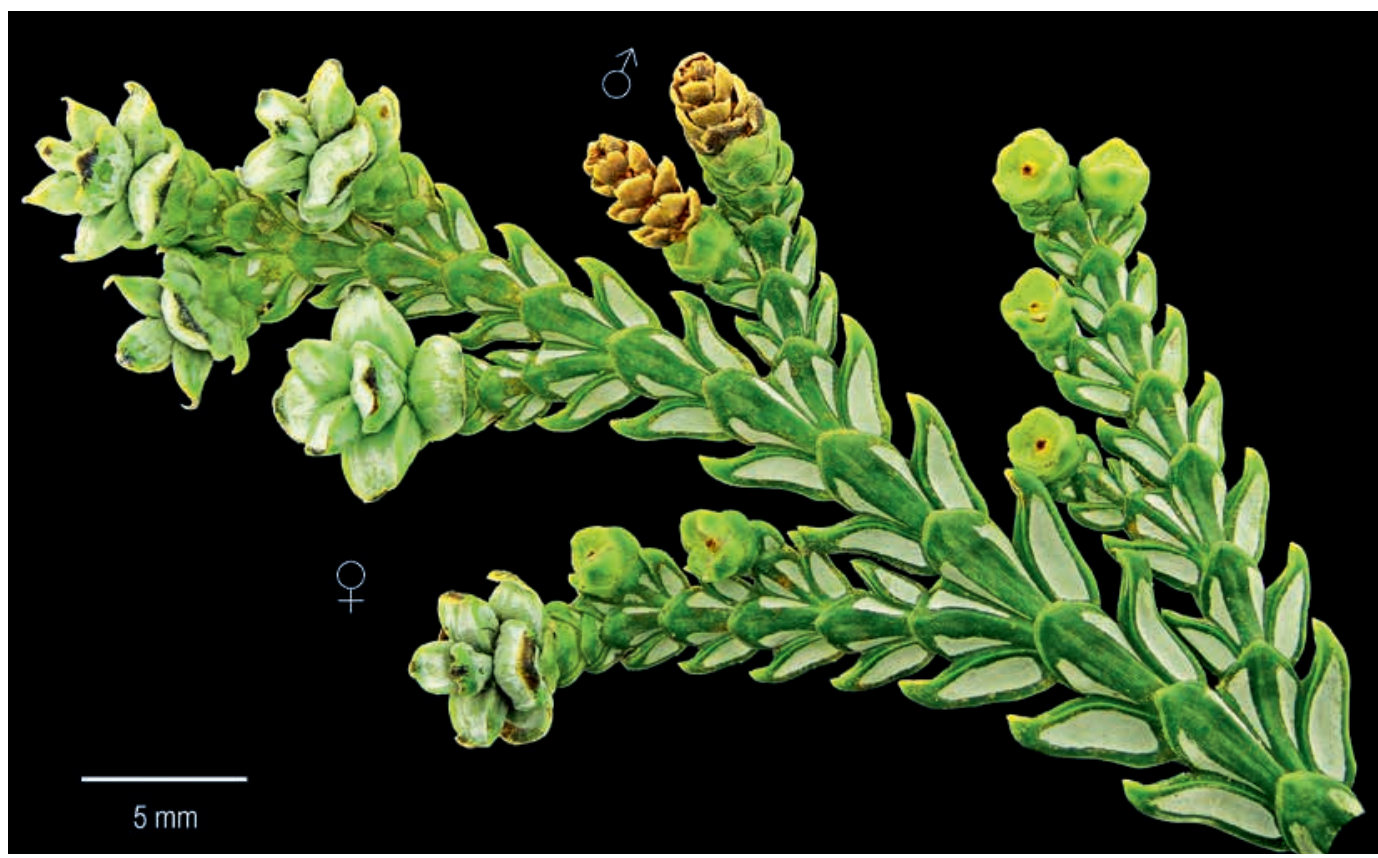

Abb. 6: Südlicher Hibalebensbaum (Thujopsis dolabrata). Männliche Zapfen mit abgespreizten Schuppen, die den Blick auf die Staubblätter freigeben. Die jungen weiblichen Zapfen heben sich nur wenig vom Blattgrün ab und sind erst später als solche zu erkennen. 


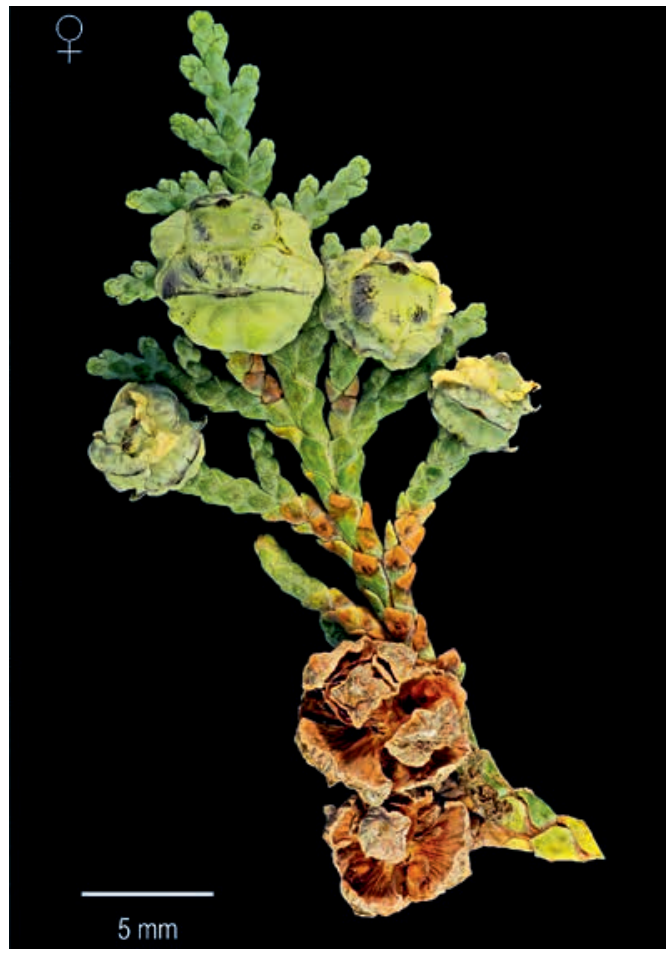

Abb. 7: Weiße Scheinzypresse (Chamaecyparis thyoides) mit kugeligen, aus mehr oder weniger sechs Schuppen aufgebauten Samenzapfen, oben heranwachsend, unten ausgereift und geöffnet.

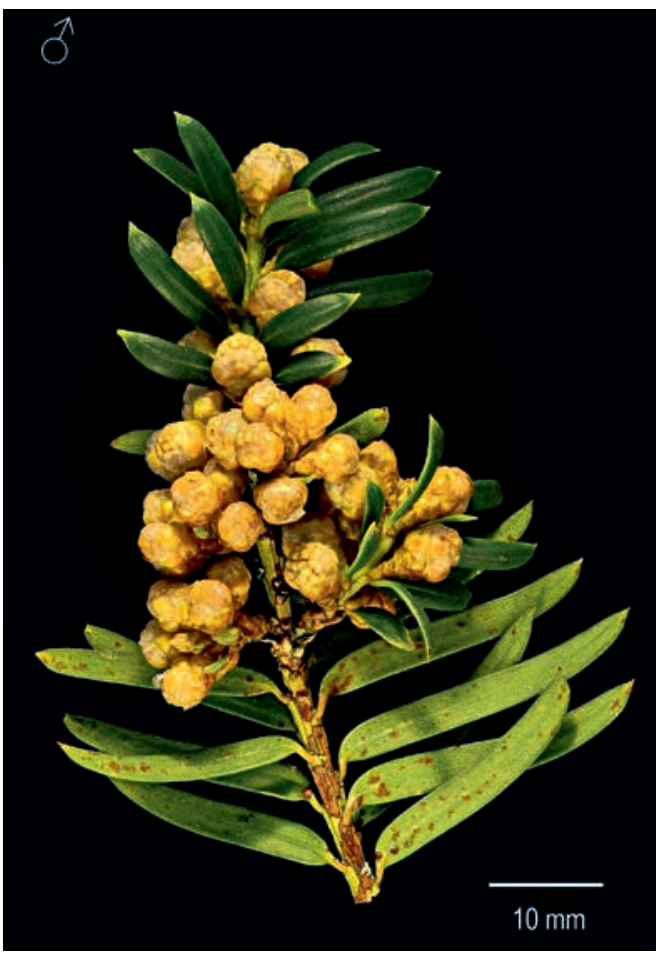

Abb. 9: Bei der Eibe (Taxus baccata) sind die Geschlechter zweihäusig verteilt.
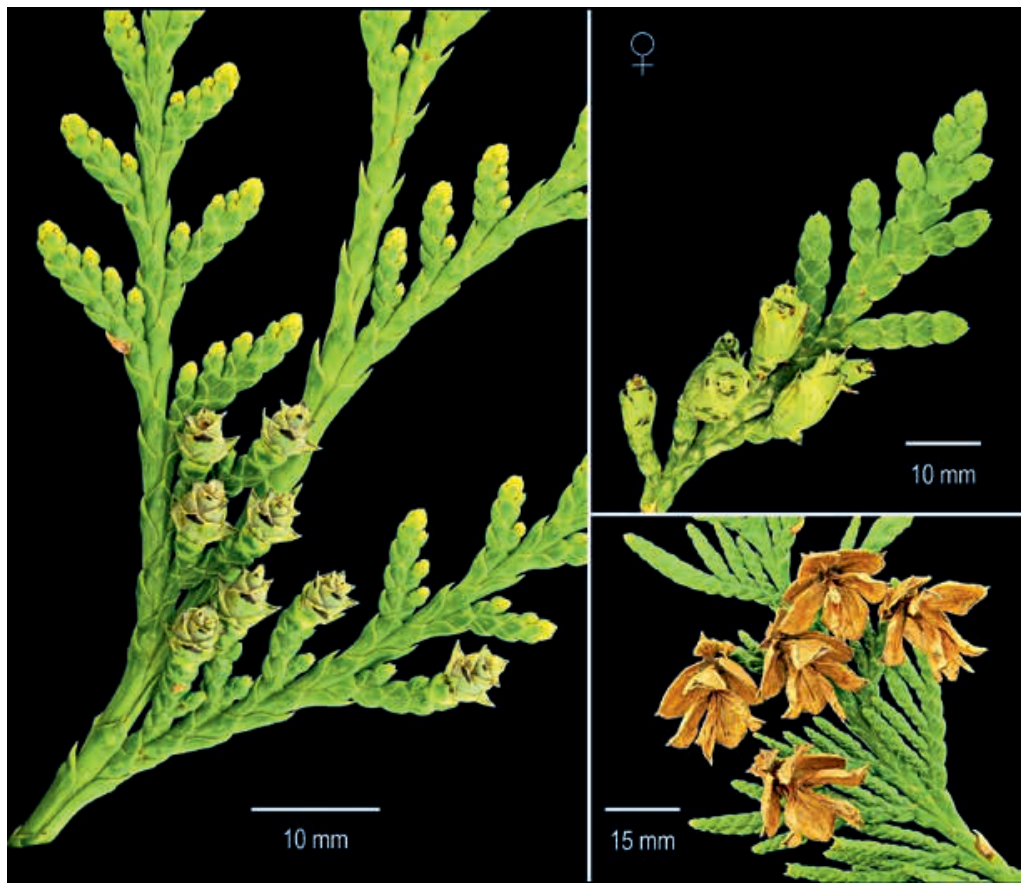

Abb. 8: Riesen-Lebensbaum (Thuja plicata); junge, sich kaum vom Zweig abhebende Zapfen (links), heranwachsende Zapfen (rechts oben), ausgereifte, geöffnete und entleerte Zapfen (rechts unten). 


\subsection{Taxaceae - Eibengewächse}

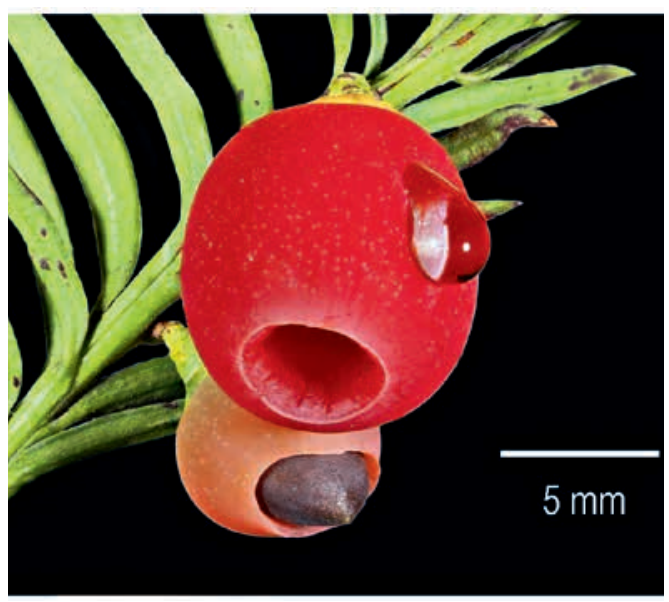

Abb. 10: Samen der Eibe (Taxus baccata) umgeben von heranreifendem (orange) bzw. ausgereiftem (rot) Samenmantel.

\subsection{Cephalotaxaceae - Kopfeibengewächse}

\section{Literatur}

DöRken, V. M. \& Nimsch, H. 2019: Morphology and identification of the world's conifer genera. - Remagen - Oberwinter.

JAGEL, A. 2011: Die Koniferen blühen - eine glitzernde Mikrowelt bei den Zypressengewächsen. - Palmengarten 75: 137-144.

Stützel, T. \& Röwekamp, I. 1997: Bestäubungsbiologie bei Nacktsamern. - Palmengarten 61: 100-109.

\section{Internetseiten}

www.focusstackingforum.de (deutsch)

www.photomacrography.net (englisch)

\section{Anschriften der Autoren}

PD Dr. Peter König, Universität Greifswald, Institut für Botanik und Landschaftsökologie, Botanischer Garten, Soldmannstr. 15, 17489 Greifswald, E-Mail: pkoenig@uni-greifswald.de

Michael Luhn, Universitätsmedizin Greifswald, Klinik für Nuklearmedizin, Fleischmannstraße 44, 17489 Greifswald, E-Mail: mluhn@uni-greifswald.de

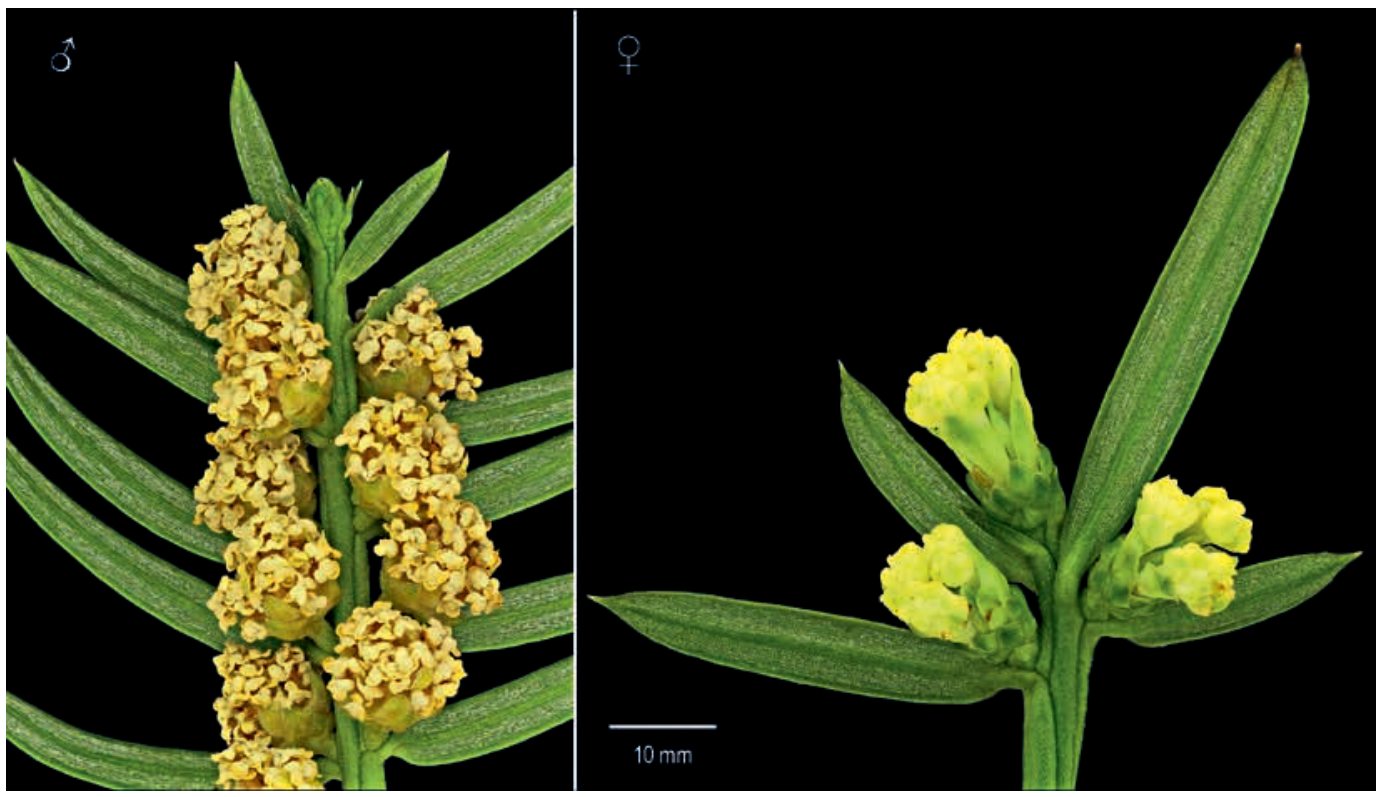

Abb. 11: Die Japanische Kopfeibe (Cephalotaxus harringtonii) ist zweihäusig. Die männlichen Zapfen sind in hängenden Köpfchen, daher der Name, angeordnet (Blick von unten im Bild links). Die weiblichen stehen in kleinen Gruppen blattachselständig; aus ihnen entwickeln sich bei der Reife auffällige Samen mit fleischiger, oliv- bis purpurner Hülle. 